\title{
Efecto del cultivo en dos pisos altitudinales sobre la síntesis de metabolitos secundarios en hojas de Stevia rebudiana Bertoni
}

\section{Effect of the cultivation in two altitudinal floors on the synthesis of secondary metabolites in Stevia rebudiana Bertoni leaves}

\author{
Bruno Giménez, María Sanabria, José Salas ${ }^{3}$
}

\section{RESUMEN}

Se determinó la existencia de diferencias significativas en la síntesis de metabolitos secundarios en hojas de Stevia rebudiana Bertoni que fueron cultivadas en dos pisos altitudinales diferentes 1.100 y $1.215 \mathrm{msnm}$, respectivamente. Los Metabolitos Secundarios (MS) fueron obtenidos por una Extracción Etanólica (EE) de las hojas de S. rebaudiana y se determinó la concentración de alcaloides, flavonoides, fenoles, saponinas y aceites esenciales. En el caso de los alcaloides la concentración fue superior a mayor altura $(1215 \mathrm{msnm})$, mientras que para los flavonoides, las saponinas y los aceites esenciales los resultaros fueron opuestos, obteniéndose la mayor concentración de estos MS a menor altura (1100msnm). Estas diferencias significativas en la concentración de los metabolitos, demuestran una vez más la plasticidad de S. rebudiana a adaptarse a ambientes heterogéneos, ajustando su fisiología y sintetizando productos naturales de elevado interés farmacológico.

Palabras claves: Metabolitos Secundarios, Stevia rebudiana, extracción etanólica (EE), farmacología.

\section{ABSTRACT}

The existence of significant differences in the synthesis of secondary metabolites in Stevia rebudiana Bertoni leaves was determined, which were cultivated in two different altitude levels 1,100 and 1,215 meters above sea level, respectively. The secondary metabolites (MS) were obtained by an Ethanolic Extraction (EE) of the leaves of S. rebaudiana and the concentration of alkaloids, flavonoids, phenols, saponins and essential oils was determined. In the case of alkaloids the concentration was higher at higher altitudes $(1215 \mathrm{msnm})$, while for flavonoids, saponins and essential oils the results were opposite, obtaining the highest concentration of these MS at lower altitudes (1100msnm). These significant differences in metabolite concentration demonstrate once again the plasticity of S. rebudiana to adapt to heterogeneous environments, adjusting its physiology and synthesizing natural products of high pharmacological interest.

Keywords: Secondary Metabolites, Stevia rebudiana, Ethanolic Extraction (EE), pharmacology.

1. Escuela de Postgrado, Universidad Alas Peruanas, Filial Ica, Perú.

2. Posgrado de Agronomía, Universidad Centroccidental Lisandro Alvarado. Tarabana, estado Lara, Venezuela.

3. Instituto Nacional de Investigaciones Agrícolas (INIA). Mérida, estado Mérida, Venezuela 


\section{INTRODUCCIÓN}

Existe a nivel global un fuerte interés creciente en el uso de edulcorantes naturales bajos en calorías que puedan funcionar perfectamente como sustituto de la glucosa en la dieta humana, la cual está siendo consumida en cantidades excesivas (Luwańska et al., 2015).

Sin embargo, la sacarosa sigue siendo el edulcorante predilecto a nivel comercial en la fabricación de diferentes bebidas de consumo masivo como jugos, refrescos, postres, yogures y diferentes tipos de alimentos en las últimas décadas (Palacio et al., 2019). Sin embargo, estudios realizados Mooradian et al., (2017) indican que el consumo excesivo de azúcar es una de las principales causas de enfermedades crónicas como la diabetes tipo II, la cual ya es considerada una epidemia a nivel mundial.

Por lo tanto, es un desafío para la industria alimentaria desarrollar nuevas formulaciones de productos con edulcorantes naturales, que estén dotados de características y sabor similar a la sacarosa y que permita mantener la calidad de sus productos (Edwards et al., 2016; Lobete et al., 2017; Carvalho et al., 2019; Kalicka et al., 2019).

Como alternativa saludable surge el uso de la stevia (S. rebudiana) por sus propiedades edulcorantes, sin calorías y es de origen natural, la cual genera beneficios a la salud en pacientes diabéticos que la utilizan como edulcorante para controlar sus niveles de glicemia en sangre y su peso corporal (Tey et al., 2017).

Esta planta es un arbusto perenne de la familia de las Asteraceae de la cual existen entre 150 a 300 especies diferentes, distribuidas a través del continente americano desde el suroeste de Estados Unidos hasta el norte de Argentina donde se le conoce popularmente como hierva dulce, hoja dulce o hoja de miel (Kumari et al., 2017).

Investigadores como Salvador-Reyes et al., (2014) demostraron experimentalmente que las hojas de stevia ha llegado a ser hasta trescientas veces más dulces que la sacarosa, de ahí que esta planta sea una alternativa para el tratamiento de enfermedades crónicas como diabetes y obesidad, pero que también puede ser consumida por personas sanas, sin efectos secundarios sobre la salud.

En su forma más sencilla son consumidas en su forma fresca sus hojas por su sabor suave y licoroso o secas y molidas, con el fin de potenciar su poder edulcorante (Millones et al., 2014).

Salvador-Reyes et al., (2014) reportaron que el consumo de stevia no incrementa los niveles de azúcar en la sangre, ya que es hipoglucémica, por el contrario mejora la tolerancia a la glucosa. Otros investigadores encontraron que el extracto metanólico de las holas de S. Rebudiana actúa como un antioxidante capaz de eliminar los radicales libres y promueve la muerte de las células cancerígenas del cuello uterino (López et al., 2016).

Por ello la importancia de caracterizar los extractos de las hojas de stevia, con la finalidad de determinar su composición química. En función de esto, Yilmaz et al., (2020) determinaron por técnicas cromatográficas en el extracto de las hojas de stevia un total de 65 compuestos orgánicos, entre ellos, los glucósidos de diterpeno, compuestos fenólicos, flavonoides, aminoácidos y ácidos grasos, independientemente de la técnica de extracción que fue utilizada.

Mientras que Kumari et al. (2017) determinaron los constituyentes químicos responsables de la dulzura, identificando a los glucósidos y dipertenoides los cuales están principalmente presentes principalmente en las hojas de la planta, siendo los principales compuestos edulcorantes el esteviósido con un sabor amargo y rebaudiósido-A sin amargura pero un 1.6-1.8 veces más dulce que el esteviósido. Una desventaja comercial es el sabor amargo que proporciona el esteviósido que limita su uso en la industria alimentaria (Formigoni et al., 2018). 
Por otro lado, la stevia también posee acción biológica y se debe principalmente a la presencia de esteviósido, esteviolbiósido, rebaudiósido A, B, C, D, E y F y dulcósido, compuestos secundarios que se localizan en las hojas de la planta en concentraciones variables, lo que sugiere que en los tejidos que constituyen este órgano (hojas), es donde ocurre principalmente la síntesis y acumulación primaria de glucósidos de esteviol de tipo diterpenoide (Vázquez et al., 2014).

Sin embargo las características de la zona donde se cultivada la planta influye directamente en la composición fisicoquímica del extracto, como lo reporta Rodríguez et al., (2010) quienes estudiaron las características físicas, químicas y sensoriales de las hojas de Stevia rebaudiana cultivada en tres tipos de paisajes: montaña, lomerío y vega; donde encontraron diferencias significativas en la concentración de los diferentes compuestos reductores, esteroles, triterpenos, glicósidos, cardiacos, saponinas y taninos dependiendo del sector donde fue cultivada la planta de stevia; como por ejemplo, el extracto acuoso obtenidos de las hojas colectadas en la zona de lomerío fueron significativamente más edulcorantes $(\mathrm{P}<0,05)$ que los extractos preparados con hojas obtenidas en las zonas de la vega y la montaña.

Por ello, estudios científicos futuros sobre la composición fisicoquímica de esta planta, serán de gran ayuda para la agroindustria, por su potencial uso al incorporarla como endulzante de bebidas y alimentos.

Sin embargo, Jarma et al., (2012) consideran que son escasos los referentes bibliográficos que documenten el efecto de las variables ambientales, sobre la síntesis diferencial de los principales glucósidos en esta planta.

Por esta razón el objetivo principal de esta investigación consistió en determinar el efecto de cultivar S. rebudiana en dos pisos altitudinales diferentes, y el efecto sobre la síntesis de metabolitos secundarios (MS) de la planta.

\section{MÉTODOS Y MATERIALES}

La investigación se realizó bajo la perspectiva del positivismo lógico y se trata de un estudio experimental, de corte transversal, donde se analizaron muestras foliares de Stevia rebudiana Bertoni recolectadas en dos pisos altitudinales diferentes, con la finalidad de determinar si existe o no de diferencias significativas en la síntesis de metabolitos secundarios en las hojas de las plantas.

Como análisis estadístico se empleó la prueba $\mathrm{t}$ de Student para muestras independientes, con la que se verifico la hipótesis del trabajo en el nivel de 0.05 de significación estadística, con el programa SPSS.

\section{A.Obtención del extracto etanólico foliar de Stevia rebaudiana Bertoni}

El material vegetal utilizado para la obtención de los extractos etanólicos (EE) foliares de S. rebaudiana consistió en hojas plenamente desarrolladas, aparentemente sanas y no senescentes, provenientes de plantas cultivadas durante 12 meses en dos pisos altitudinales diferentes, el primero se ubicó en las instalaciones del Instituto de Investigaciones Agrícolas (INIA), en San Juan de Lagunillas, a $1.100 \mathrm{msnm}$ y el segundo en Santa Catalina a $1.215 \mathrm{msnm}$, ambas localidades en el estado Mérida-Venezuela.

Las hojas de S. rebaudiana fueron seleccionadas de 30 plantas adultas (15 de cada localidad), y secadas a la sombra. Se pesaron por separado $250 \mathrm{~g}$ de hojas secas de cada planta, para luego proceder a molerlas en una licuadora convencional OsterMR, el polvo se maceró en $120 \mathrm{~mL}$ de alcohol etílico $\left(96^{\circ}\right)$ en frascos de vidrios color ámbar.

Después de 12 horas se filtró la mezcla, el líquido obtenido se destilo con la ayuda de un Rotovapor Buchi 461 Watter Bath para obtener el EE, el que fue almacenado en tubos de ensayo a $10^{\circ} \mathrm{C}$, hasta el momento de realizar las pruebas para la determinación y cuantificación de los principales grupos de MS (Marcano y Hasegawa, 2002). 
B.Determinación y cuantificación de metabolitos secundarios (MS) en los extractos etanóli$\cos (\mathrm{EE})$ foliares de Stevia rebaudiana Bertoni.

Para determinar la presencia de alcaloides, flavonoides y fenoles en el EE obtenido de las hojas de S. rebaudiana se utilizó la cromatografía de capa fina de tipo ascendente con cromatofolios de silica gel MERK MR y en cada uno se dispersaron con la ayuda de una micropipeta 2 gotas, equivalentes a $5 \mu \mathrm{L}$ cada una (para un total de $10 \mu \mathrm{L}$ ) a $0,6 \mathrm{~cm}$ de la base.

En una cámara cromatografía, que contenía el eluyente específico para cada grupo de MS, am- bos se dejaron ascender hasta los 0,7 cm antes del borde superior del cromatofolio, se retiró y se dejó secar para luego, realizar el revelado respectivo, según el grupo de compuesto secundario que se deseaba determinar.

Los eluyentes utilizados, la proporción de los componentes de cada uno y el método de revelado de la cromatografía se muestran en el cuadro 1 (Marcano y Hasegawa, 2002).

Tabla $\mathrm{N}^{\circ} 1$. Eluyentes y métodos de revelados para la determinación de alcaloides, flavonoides y fenoles en extractos etanólicos (EE) foliares de Stevia rebaudiana Bertoni.

\begin{tabular}{|c|c|c|c|c|}
\hline MS & \multicolumn{2}{|c|}{$\begin{array}{c}\text { Eluyente y relación } \\
(\mathrm{ml})\end{array}$} & Revelado & Resultados \\
\hline Alcaloides & $9: 2: 1$ & $\begin{array}{l}\text { N-Butanol, ácido } \\
\text { acético y agua }\end{array}$ & $\begin{array}{l}\text { Lámpara de luz } \\
\text { ultravioleta (UV }\end{array}$ & $\begin{array}{l}+ \text { Coloración } \\
\text { amarillo- } \\
\text { anaranjado }\end{array}$ \\
\hline Flavonoides & $6: 3: 5: 1$ & $\begin{array}{l}\text { Benceno, ácido } \\
\text { acético y agua }\end{array}$ & & $\begin{array}{l}+ \text { Coloración } \\
\text { fluorescente } \\
\text { blanca }\end{array}$ \\
\hline Fenoles & $9: 1$ & $\begin{array}{c}\text { Agua y ácido } \\
\text { acético }\end{array}$ & $\begin{array}{c}\text { Cloruro férrico } \\
(1 \%)\end{array}$ & $\begin{array}{l}+ \text { Coloración } \\
\text { férrica. }\end{array}$ \\
\hline
\end{tabular}

C. Determinación de aceites esenciales en el extracto etanólico (EE) de las hojas de S. rebaudiana Bertoni.

Para la determinación de la presencia o no de aceites esenciales se agitó $1 \mathrm{~mL}$ del $\mathrm{EE}$ en un tubo de ensayo, y a través del olfato se evidenció la presencia o no del aroma característico que le confieren este grupo de compuestos secundarios al mismo (Rodríguez, 2007).

Posteriormente se obtuvieron por hidrodestilación colocando $100 \mathrm{~g}$ de hojas de estevia de cada una de las localidades, por separado, en un balón hidrodestilando por 45 min y se midió el volumen de estos compuestos secundarios obtenidos con la ayuda de una micropipeta. El resultado se expresó en $\mathrm{mL}$ de aceites obtenidos por $100 \mathrm{~g}$ de hoja seca $(\mathrm{mL} \cdot 100 \mathrm{~g}-1)$, descontando la humedad residual (World Health Organization, 1998).

D. Determinación de saponinas en el extracto etanólico (EE) foliares de S. rebaudiana Bertoni.

Para la determinación de la presencia de saponinas, el extracto fue diluido en agua destilada (1:1) $\mathrm{y}$ agitado vigorosamente durante $1 \mathrm{~min}$ hasta la 
formación de espuma, cuya persistencia después de $15 \mathrm{~min}$, fue considerada positiva para saponinas. El contenido de saponinas fue valorado de acuerdo con la escala de Cuéllar et al. (1999) la cual se explica a continuación: $0 \mathrm{~mm}$ (negativo); 0,1-5,0 mm (muy bajo); 5,1-9,0 mm (bajo); 9,1$14 \mathrm{~mm}$ (moderado) y $>14 \mathrm{~mm}$ (alto).

\section{E. Cuantificación de metabolitos secundarios en extractos el etanólico (EE) de las hojas de Stevia rebaudiana Bertoni.}

Para determinar la concentración de los grupos de MS considerados, se siguió la metodología de Vásquez et al. (2008). Esta consistió en marcar con la ayuda de un lápiz de grafito el área ocupada por la presencia del grupo de MS en el cromatofolio de sílica/gel.

Posteriormente se extrajeron tres circunferencias de área conocida $(\mathrm{AC})$ con ayuda de un perforador, cada una fue raspadas y pesadas en una balanza analítica (Ohaus Adventure $\mathrm{N}^{\circ}$ AR2140) para determinar el peso (g) del MS + la sílica/gel + el eluyente.

De manera similar, el espacio restante del área recorrida por el MS, constituido por MS + síli$\mathrm{ca}+$ solvente, fue raspado y denotado como área desconocida ( $\mathrm{AD})$. Adicionalmente se preparó un número similar de discos de cromatofolios conteniendo solo sílica + solvente, los que constituyeron el control. Estos discos también fueron raspados y pesados para obtener el área de control (ACt).

La cantidad total de MS (expresada en $\mathrm{mg} / \mathrm{mL}$ ) contenidas en la mancha del cromatofolio fue calculada mediante la siguiente fórmula:

$$
\mu g d e M S / m L=\frac{\left[\left(A D *\left(\frac{A C-A C t}{A C}\right)+(A C-A C t)\right) * 1000\right]}{10 \mu L}
$$

\section{RESULTADOS}

En el cuadro 2 se muestran los resultados obtenidos de la determinación y cuantificación de los grupos de MS en el EE foliar de la S. rebudiana, las cuales fueron cultivadas a dos pisos altitudinales diferentes 1.100 y $1.215 \mathrm{msnm}$, respectivamente; en las localidades ubicadas en el Estado Mérida, Venezuela.

Se determinó la presencia de alcaloides, flavonoides, aceites esenciales y saponinas. Los fenoles totales resultaron negativos.

Tabla $\mathrm{N}^{\circ}$ 2. Valores promedios de la cuantificación de los grupos de metabolitos secundarios en extractos etanólicos (EE) foliares de plantas de Stevia rebudiana Bertoni, cultivadas en dos pisos altitudinales.

\begin{tabular}{ccccc}
\hline $\begin{array}{c}\text { Piso } \\
\text { altitudinal }\end{array}$ & $\begin{array}{c}\text { Alcaloides } \\
\mathrm{mg} \cdot \mathrm{mL} \text { de } \mathrm{EE}\end{array}$ & $\begin{array}{c}\text { Flavonoides } \\
\mathrm{mg} \cdot \mathrm{mL} \text { de EE }\end{array}$ & $\begin{array}{c}\text { Saponinas } \\
\mathrm{mm}\end{array}$ & $\begin{array}{c}\text { Aceites esenciales } \\
\mathrm{mL} 100 \mathrm{~g}^{-1}\end{array}$ \\
\hline 1.100 & $6,94 \pm 0,03$ & $1,31 \pm 0.06$ & $2,90 \pm 0.07$ & $10,5 \pm 0.08$ \\
1.215 & $3,06 \pm 0,05$ & $2,09 \pm 0.04$ & $3,70 \pm 0.04$ & $15,4 \pm 0.07$ \\
& $\mathrm{p}=0.000$ & $\mathrm{p}=0.000$ & $\mathrm{p}=0.000$ & $\mathrm{p}=0.000$ \\
\hline
\end{tabular}

Unidades: m.s.n.m.=metros sobre el nivel del mar; $\mathrm{mg} \cdot \mathrm{mL}$ de $\mathrm{EE}=$ miligramos de $\mathrm{MS}$ por mililitro de extracto acuoso; $\mathrm{mm}=$ milímetros, $\mathrm{x}^{\prime}=$ media, $\mathrm{s}=$ desviación estándar, $\mathrm{p}=$ significancia. 
Como lo indica la tabla 2, se puede observar una diferencia significativa $(\mathrm{p}<0.005)$ en la concentración de alcaloides, obteniendose mayores concentraciones de este grupo de compuestos nitrogenados en los extractos etanólicos de las hojas de stevia cultivadas a $1100 \mathrm{msnm}$ que las cultivadas a mayor altura $1215 \mathrm{msnm}$.

Efecto contrario el observado para los flavonoides, las saponinas y los aceites esenciales, donde como se puede observar en la tabla 2 existe una diferencia significativa $(\mathrm{p}<0.005)$ en las concentraciones de estas especies analizadas en el extracto etanólico de las hojas de stevia cultivadas a mayor altura $1215 \mathrm{msnm}$ que a $1100 \mathrm{msnm}$.

Adicionalmente, en el caso de las saponinas al comparar los resultados obtenidos con las concentraciones establecidas por Cheok et al., (2014) donde propone que valores entre 9,1-14 $\mathrm{mm}$ son consideradas cantidades moderadas de saponinas, y valores superiores a $14 \mathrm{~mm}$ son altos, los resultados indican que a $1100 \mathrm{msnm}$ los contenidos de saponinas en el extracto etanólico de las hojas de stevia se consideran como moderadas, mientras que a $1215 \mathrm{msnm}$, el contenido es alto.

\section{DISCUSIÓN}

De los resultados obtenidos en esta investigación se pudo inferir que los pisos altitudinales a los cuales fueron cultivadas las plantas de stevia, tuvieron un efecto significativo sobre la síntesis de metabolitos secundarios.

Tal como lo señalaron Jarma et al., (2012) los glucósidos de esteviol son sintetizados en la ruta de las giberelinas, por tanto factores tales como la luz, la temperatura y en el caso de esta investigación, la altitud, también tienen un efecto importante sobre la síntesis de los mismos. Una situación semejante fue evidenciada por Ahmad et al., (2016) cuando observaron que el tipo y la intensidad de la luz mejoran significativamente la producción de metabolitos secundarios antioxidantes en la lanta de stevia.
Las diferencias significativas observadas en cuanto a la síntesis de un grupo de MS en la planta cultivadas a 1.100 y $1.215 \mathrm{msnm}$ se podrían explicar por el hecho de que está influenciada por la dinámica de las interacciones entre estas y su ambiente biótico y abiótico (A., González-Paleo, \& Ravetta, 2011). Así mismo, (Cheok et al., 2014) consideraron que como parte de la protección química, una de las estrategia utilizada por el vegetal para su protección, es la producción de estos compuestos con actividad antimicrobiana, es contra de herbívoros o como antioxidante.

En el caso de los EE obtenidos a partir de las hojas de plantas de S. rebudiana, la variación fue cuantitativa y no en cuanto a grupos de MS, lo cual debía esperarse por tratarse de plantas taxonómicamente iguales y según Santacoloma-Varón, et al., (2010) la existencia de una gran variación cualitativa y cuantitativa en el contenido de estos compuestos es más común entre diferentes especies vegetales y el contenido de estas sustancias puede alcanzar hasta un $40 \%$ de la materia seca dependiendo de factores climáticos, edafológicos y del órgano de la planta que se considere. Aunado a esto, estos mismos autores encontraron que los distintos compuestos que puede producir una especie presentan una determinada distribución dentro de los órganos, tejidos y células de una planta y ello responde frecuentemente a las influencias ambientales.

La presencia de las saponinas, en los extractos obtenidos de las plantas de stevia en los dos pisos altitudinales, era de esperarse también ya que estas biomoléculas, en forma glucosídica, actúan como esteroides reguladores del crecimiento y pueden generar en las plantas un aumento en el crecimiento y desarrollo, además de una rápida recuperación, luego de la poda, mediante la presencia de brotes abundantes (Cheok et al., 2014).

Este grupo de compuestos pudo haber influenciado que las plantas que durante su desarrollo inicial no tenían ramificaciones, se transformaran en multicaule después de la primera cosecha y según Quezada-Nieves, (2008), pueden llegar a 
formar en los primeros años hasta un promedio de veinte tallos.

El caso de los aceites esenciales, la concentración fue tres veces superior en los EE de las hojas de las plantas cultivadas a $1.215 \mathrm{msnm}$. Según investigaciones previas, la composición química de este grupo de compuestos de las diferentes especies de stevia ha sido muy estudiada y es muy variada, existiendo la predominancia de borneol, cineol, pulegona, geraniol, nerol y limoneno, los cuales han sido señalados en estudios previos principalmente para S. satureiaefolia, muy común en Argentina (Pérez-Pérez, 2006).

Los alcaloides, flavonoides, saponinas y aceites esenciales, detectados en los extractos de stevia utilizados en esta investigación, junto con los fenoles, tienen una gran importancia ecológica, ya que estos compuestos participan en los procesos de adaptación de las plantas al ambiente, así como también se induce una síntesis activa de los mismos cuando el vegetal es expuesto a condiciones adversas, en el caso a dos pisos altitudinales diferentes, por lo que se podría inferir que las mismas estuvieron bajo condiciones de estrés abiótico (Nieto-Ramírez et al., (2018).

Los resultados obtenidos en esta investigación, permiten inferir que tal como lo señaló Ávalos y Pérez, (2009) las plantas de stevia pudiesen haber destinado una cantidad significativa del carbono asimilado y de la energía a la síntesis de moléculas orgánicas o productos secundario que tienen funciones ecológicas específicas, en este caso protegiéndolas de situaciones que pudiesen ser estresantes para las mimas.

\section{CONCLUSIONES}

Con base a estos resultados se puede concluir que los pisos altitudinales tuvieron un efecto sobre la síntesis de alcaloides, flavonoides, saponinas y aceites esenciales.

Los resultados obtenidos no sólo contribuyen al conocimiento de los compuestos secundarios presentes en los extractos foliares particularmente de S. rebudiana cultivadas a dos pisos altitudinales, sino que también son de gran utilidad a la hora de incrementar la concentración de los grupos de los metabolitos secundarios responsables de las propiedades biológicas del vegetal.

Las diferencias en cuanto a la concentración de los grupos de compuestos, demuestran una vez más la plasticidad de S. rebudiana a adaptarse a ambientes heterogéneos, ajustando su fisiología, sintetizando productos naturales de elevado interés farmacológico.

\section{REFERENCIAS BIBLIOGRÁFICAS}

A., V., González-Paleo, L., \& Ravetta, D. (2011). Metabolismo secundario de plantas leñosas de zonas áridas: mecanismos de producción, funciones y posibilidades de aprovechamiento. Ecología Austral, 2(3), 317-327. Recuperado de http://hdl.handle.net/20.500.12110/ecologiaaustral_v021_n03_p317

Ahmad, N., Rab, A., \& Ahmad, N. (2016). Light-induced biochemical variations in secondary metabolites production and antioxidant activity in callus cultures of Stevia rebaudiana (Bert.). Research article for Journal of Photochemistry and Photobiology B: Biology, 54, 51-56. https://doi. org/https://doi.org/10.1016/j.jphotobiol.2015.11.015

Ávalos, A., \& Pérez, E. (2009). Metabolismo secundario de plantas. Reduca, 2(3), 119-145. Recuperado de https:// eprints.ucm.es/9603/1/Metabolismo_secundario_de_ plantas.pdf

Carvalho, M. W., Arriola, N. D. A., Pinto, S. S., Verruck, S., Fritzen-Freire, C. B. Prudêncio, E. S., \& Amboni, R. D. M. C. (2019). Stevia-fortified yoghurt: stability, antioxidant activity and in vitro digestion behavior. International Journal of Dairy Technology, 72(1), 57-64. https://doi.org/ http://dx.doi.org/10.1111/1471-0307.12559

Cheok, C. Y., Abdel, H., Salman, K., \& Sulaiman, R. (2014). Extraction and quantification of saponins : A review. Food Research Internationa, 59, 16-40. https://doi.org/http://dx. doi.org/10.1016/j.foodres.2014.01.057

Edwards, C. H., Rossi, M., Corpe, C. P., Butterworth, P. J., \& Ellis, P. R. (2016). The role of sugars and sweeteneers in food, diet and health: alternatives for the future. Trends in Food Science \& Technology, 56, 158-166. https://doi.org/ http://dx.doi.org/10.1016/j.tifs.2016.07.008 
Formigoni, M., Milani, P. G., Silva-Avíncola, A., Santos, V. J., Benossi, L., Dacome, A. S., ... Costa, S. C. (2018). Stevia rebaudiana leaves pretreated with ethanol and characterization of the ethanolic extract by UPLC-HRMS. Food Chemistry, 241, 452-459. https://doi.org/http://dx.doi.org/10.1016/j.foodchem.2017.09.022

Jarma, O., Cardona, A., \& Fernández, C. (2012). Efecto de la temperatura y radiación en la producción de glucósidos de esteviol en Stevia rebaudiana en el Caribe húmedo colombiano. Revista U.D.C.A Actualidad \& Divulgación Científica, 15(2), 339 - 347. Recuperado de http:// www.scielo.org.co/scielo.php?script=sci_arttext\&pi$\mathrm{d}=$ S0123-42262012000200011

Kalicka, D., Znamirowka, A., Pawlos, M., Buniowska, M., \& Szajnar, K. (2019). Hysical and sensory characteristics and probiotic survival in ice cream sweetened with various polyols. International Journal of Dairy Technology, 72, 456-465.

Kumari, N., Rana, R. C., Sharma, Y. P., \& Kumar, S. (2017). Extraction, purification and analysis of sweet compounds in Stevia rebaudiana bertoni using chromatographic techniques. Indian Journal of Pharmaceutical Sciences, 79(4), 617-624. https://doi.org/10.4172/pharmaceutical-sciences. 1000270

Lobete, M. M., Baka, M., Noriega, E., Jooken, E., Monballiu, A., De Beurme, S., ... Van Impe, J. F. (2017). Stevia-based sweeteners as a promising alternative to table sugar: the effect on Listeria monocytogenes and Salmonella Typhimurium growth dynamics. International Journal of Food Microbiology, 245, 38-52. https://doi.org/http://dx.doi.org/10.1016/j.ijfoodmicro.2017.01.008

López, V., Pérez, S., Vinuesa, A., Zorzettoc, C., \& Abianbd, O. (2016). Stevia rebaudiana ethanolic extract exerts better antioxidant properties and antiproliferative effects in tumour cells than its diterpene glycoside stevioside. Food Funct., 7, 2107-2113. https://doi.org/https://doi. org/10.1039/C5FO01586C

Luwańska, A., Perz, A., Mańkowska, G., \& Wielgus, K. (2015). Application of in vitro stevia (Stevia rebaudiana Bertoni) cultures in obtaining steviol glycoside rich material. Herba Polonica, 61(1), 50-63. https://doi.org/10.1515/ hepo-2015-0010

Millones, C., Mori, G., Bacalla, J., Vásquez, E., \& Tafur, R. (2014). Obtención de un filtrante de anís de monte (Tagetes filifolia Lag.) edulcorado con hojas de estevia (Stevia rebaudiana Bertoni). Scientia Agropecuaria, 5(1), 45-51. Recuperado de http://www.scielo.org.pe/pdf/agro/v5n1/ a05v5n1.pdf
Mooradian, A. D., Smith, M., \& Tokuda, M. (2017). The role of artificial and natural sweeteners in reducing the consumption of table sugar: A narrative review. Clinical Nutrition ESPEN, 18, 1-8. https://doi.org/https://doi.org/10.1016/j.clnesp.2017.01.004

Nieto-Ramírez, M. I., García-Trejo, J. F., Caltzontzin-Rabell, V., Chávez-Jaime, R., \& Estrada-Sánchez, M. de la L. (2018). Efecto de las condiciones de cultivo en la producción de fenoles, flavonoides totales y su capacidad antioxidante en el árnica ( Heterotheca inuloides ). Revista Mexicana de Ciencias Agrícolas, 21, 4296-4305. Recuperado de http://www.scielo.org.mx/pdf/remexca/v9nspe21/20070934-remexca-9-spe21-4296.pdf

Pérez-Pérez, I. (2006). Metabolitos secundarios aislados de hojas y raíces de Stevia jorullensis (Instituto de Ciencias Básicas e Ingeniería). Recuperado de https://repository. uaeh.edu.mx/bitstream/handle/123456789/10677

Quezada-Nieves, F. (2008). Propagación por esquejes de Stevia (Stevia rebaudiana Bert) en tres sustratos y dos dosis de hormona de enraizamiento bajo invernadero en el Cantón Santa Isabel (Universidad de Cuenca). Recuperado de http://dspace.ucuenca.edu.ec/bitstream/123456789/3032/1/tag294.pdf

Rodríguez, W., Perdomo, D., Sánchez, I., Rosas, G., Dolores, C., \& Bedoya, C. (2010). Caracterización física, química y sensorial de la hoja de Stevia rebaudiana (var. morita II) cultivada en tres paisajes del Caquetá (Colombia). Momentos de Ciencia, 7(1), 57-65.

Salvador-Reyes, R., Sotelo-Herrera, M., \& Paucar-Menacho, L. (2014). Estudio de la Stevia (Stevia rebaudiana Bertoni) como edulcorante natural y su uso en beneficio de la salud. Scientia agropecuaria, 5, 157-163. https://doi. org/10.17268/sci.agropecu.2014.03.06

Tey, S. L., Salleh, N. B., Henry, J., \& Forde, C. G. (2017). Effects of aspartame-, monk fruit-, stevia- and sucrose-sweetened beverages on postprandial glucose, insulin and energy intake. Int J Obes, 41, 450-457. https://doi.org/ https://doi.org/10.1038/ijo.2016.225

Vázquez-Baxcajay, L., Robledo-Paz, A., Muratalla-Lúa, A., \& Conde-Martínez, V. (2014). MICROPROPAGACIÓN DE Stevia rebaudiana Bertoni Y DETECCIÓN DE STEVIÓSIDOS. Bioagro, 26(1), 49-56. Recuperado de http:// www.redalyc.org/articulo.oa?id=85730396006

Yılmaz, F. M., Görgüç, A., Uygun, Ö., \& Bircan, C. (2020). Steviol glycosides and polyphenols extraction from Stevia rebaudiana Bertoni leaves using maceration, microwave-, and ultrasound-assisted techniques. SEPARATION SCIENCE AND TECHNOLOGY. https://doi.org/10.1080 /01496395.2020.1743311 